\title{
Weltraumastronomie an der Wiener Universitäts-Sternwarte
}

\author{
Anneliese Schnell und Werner W. Weiss \\ Institut für Astronomie, Türkenschanzstrasse 17, 1180 Vienna, Austria
}

\begin{abstract}
An overview on various activities in space astronomy carried out at the Institute for Astronomy of the University of Vienna is given.
\end{abstract}

Die Einstellung gegenüber der Weltraumastronomie war an der Wiener Universitäts-Sternwarte, wie in vielen anderen klassischen astronomischen Instituten, in der Zeit vor dem Start der Sputniks nicht gerade positiv. Begründet wurde das damit, dass man über Planeten alles wisse, dass es keine stabilen Satellitenbahnen gäbe, dass auf dem Mond zu viel Staub vorhanden wäre, in dem Astronauten versinken würden, und dass - wie J. Hopmann meinte - die Strahlung die Astronauten ohnehin umbringen würde.

Bemerkenswerterweise galt aber eine der Forschungsinteressen Hopmanns dem Mond, während seiner Zeit als Direktor der Wiener Sternwarte (1951 - 1958) beschäftigte er sich, zum Teil gemeinsam mit Guntram SchrutkaRechtenstamm, mit der Figur des Mondes, mit der Bestimmung selenographischer Koordinaten und mit Messungen von Höhen auf dem Mond (Ferrari d'Occhieppo 1977 mit Schriftenverzeichnis, Haupt 1996). G. Schrutka bestimmte 1958 Koordinaten von 150 Punkten auf dem Mond durch eine Neureduktion der Breslauer Messungen von J. Franz unter Verwendung einer neuen Librationstheorie und neuer Librationskonstanten. Diese Koordinaten - die damals genauesten publizierten - wurden bei der Erstellung einer Topographischen Mondkarte zur Vorbereitung der ersten amerikanischen Mondmissionen benutzt (DMAAC 1973 = Defense Mapping Agency Aerospace Center Febr. 1973). In dieser Zeit wurden auch Dissertationsthemen aus dem Gebiet der Vermessung des Mondes vergeben; z.B. die Ableitung relativer Mondhöhen (Swaton 1965). Hopmann nahm an mehreren Mondkonferenzen teil, wo er über die Ergebnisse der Wiener Arbeiten berichtete. Ebenso wurde er von der 


\section{4,1.7 LUTAR RARTESIDB MAPS \\ (Published by USATOPOCOY)}

Topograph1c Lunar Map

1st Bd1tion, September 1964

Scale: $1: 5,000,000$

Projection Modified Stereographic

Limits: $90^{\circ} \mathrm{H}-\mathrm{S}, 180^{\circ}$

Number of Sheets: 2

Sheet s1ze: $54^{\prime \prime} \times 38^{\prime \prime}$

Topograph1c Lunar Map

1st Bdition, June 1965

Scale: $1: 2,000,000$

Projection Modifled Stereographic

Limits : $90^{\circ} \mathrm{N}-\mathrm{S}, 180^{\circ}$

Number of Sheets: 6

Sheet Size: $54^{\prime \prime} \times 38^{\prime \prime}$

Lunar earthside maps were produced to provide full topographic aap coverage pending the avallability of spacecraft photography of the moon, and to assist in the planning of early lungr missions such as those of the Ranger and Orbiter aeries. These maps were produced in separate Shaded Relief, Rellef, and Gradient Tint versions. The contour interval is 1000 meters with oupplementary contours at 500 meter intervalo. Relief is shown by form lines in some areas.

Bight palrs of photographs taken at Parls Observatory between Karch 1896 and January 1907 were used as compllation source. The photographs of each pair were taken near the extreme long1tude 11bratione of $\pm 7^{\circ} 54^{\prime}$. The pairs of photoв were thus stereo pairs with an effective baseine of about 65,000 miles. The acale of the source photography is about $1: 22,000,000$.

The control used in the compilation of these mapa consigted of the coordinates of 150 points determined by Schrutka-Rechtenstamm in 1958 described in Section 3.1.2. The horizontal datum was the crater Mosting A, which was assigned latitude $3^{\circ} 10^{\prime} 47^{\prime \prime}$ south and longitude $354^{\circ} 50^{\prime} 13^{\prime \prime}$, bsaed on Schrutka-Rechtenstamm control. Mosting A was aloo chosen as the vertical datum. However, it vas considered desirable to avoid the use of negative elevation values. As the crater Ariatarchus was found to be the lowest feature in the control system, $7.0 \mathrm{k} 1$ lometers lower than Мовting $\mathrm{A}$, it was aselgned an elevation of zero, taking the elevation of the vertical datum 7000 meters.

Section 4.1 .7

1

Abb. 1: Begleittext zu DMAAC 1973

sowjetischen Akademie der Wissenschaften zu Vortragsreisen eingeladen. Die nachfolgende Generation Wiener Astronomen war mehr an stellaren Phänomenen und an Datenverarbeitung interessiert. Magnetische Sterne wurden zu 
einem wichtigen Arbeitsgebiet am Institut. So wurden Ultraviolettbeobachtungen von alpha And mit dem Satelliten TD-1 der ESRO (Rakos, Kamperman 1977) durchgeführt. Spektren von alpha And wurden auch mit dem Copernicus (OAO3) Satelliten erhalten. Und natürlich wurde umfangreiches Beobachtungsmaterial mit dem International Ultraviolet Explorer IUE gewonnen, wobei die Wiener Astronomen beide Bodenstationen (Villafranca und Goddard) nutzten.

Die jungen Kollegen, die in den Siebzigerjahren des 20. Jahrhunderts studiert hatten, waren auch im Umgang mit Computern geschult, sowie in der Reduktion von Daten. So wurde 1980 in Wien gemeinsam mit der Österreichischen Akademie der Wissenschaften und der Austrian Solar and Space Agency, ASSA, ein "IUE Data Reduction Workshop" mit 54 Teilnehmerinnen und Teilnehmern vor allem aus den ESA Mitgliedstaaten organisiert (Weiss, Schnell, Albrecht, Jenkner, Maitzen, Rakos, Mondre 1980). Bei einem 1978 abgehaltenen Workshop "Ap-Stars in the Infrared" (Weiss, W. W., Kreidl, T. J. 1978) wurde weiters über die Verwendung von Satellitendaten im Infrarot diskutiert.

Sowohl Institutsmitglieder als auch Studierende nahmen regelmäßig an den von der ASSA veranstalteten Sommerschulen zu Weltraumthemen in Alpbach teil.

Bereits ab 1977 gehörte R. Albrecht dem ESA Science Instrument Team für die Faint Object Camera des Hubble Space Telescope an, dann auch dem Instrument Science and Software Team. Er arbeitete schließlich zunächst während einiger Forschungsaufenthalte in den USA und ab 1983 endgültig bei der ESO in Garching als Deputy Head der European Coordinating Facility des Hubble Space Telescope. Ein anderer Absolvent, H. Jenkner, verliess das Institut 1984 um am Hubble Space Telescope Science Institute in Baltimore zu arbeiten, wo er jetzt die Abteilung Hubble Legacy leitet.

1978 wurde von K. Rakos ein Area Scanner für das Spacelab mit dem Ziel der Bestimmung der Wellenlängenabhängigkeit der Abplattung der Sonne konzipiert. 1980 wurden weiters Überlegungen angestellt, ob sich die Wiener Sternwarte als "Host Institute" für die European Coordinating Facility für das Hubble Space Telescope bewerben solle. Allerdings wurden beide Projekte nicht realisiert.

Bei dem 1978 in Wien veranstalteten IAU Colloquium No. 48 über "Modern Astrometry" wurde die Bedeutung von Satellitendaten in der Astrometrie vor allem in Bezug auf Messgenauigkeit und auf Vergrößerung des vorhandenen Datenmaterials diskutiert (Prochazka F. V., Tucker, R. H., 1978).

Ein von ESA, ESO und ASSA 1986 veranstalteter Workshop "Interrelation of Ground Based and Space Astronomy" bedeutete den Beginn der Weltraumaktivitäten für eine weitere Arbeitsgruppe der Sternwarte, die sich mit Spätstadien der Sternentwicklung beschäftigte. Vor allem J. Hron und F. Kerschbaum begannen sich daraufhin für das Infrared Space Observatory, ISO, zu interessieren. 
ISO wurde schließlich von der ESA bei einer internationalen Pressekonferenz 1996 auch in Wien vorgestellt.

R. Albrecht unterstützte Mitarbeiter des Instituts für Sportwissenschaften bei der Datenauswertung des Experiments MOTOMIR, das vom österreichischen Kosmonauten F. Vieböck ausgeführt wurde. Dieses Experiment war Teil des ersten (und letzten) österreichischen bemannten Weltraumprojektes, AUSTROMIR, das in Kooperation mit der damaligen Sowjetunion auf der MIRStation stattfand.

Den Mitgliedern der Arbeitsgruppe "Sternatmosphären und pulsierende Sterne" erschien eine Teilnahme am Projekt EVRIS (Étude de la Variabilité, de la Rotation et des Interieurs Stellaires) von besonderer Bedeutung. Dieser Satellit sollte in einer Zusammenarbeit zwischen Frankreich, Österreich und Russland sonnenähnliche Pulsationen in Sternen nachweisen. Erstmals diskutiert wurde das Projekt während des IAU Kolloquiums Nr. 137 "Inside the Stars" September 1992 in Wien (Baglin, A., Weiss, W. W., Bisnovatyi-Kogan, G. 1993). Die intensiven Vorbereitungsarbeiten werden auch durch die Vergabe von Themen z. B. für Diplomarbeiten demonstriert (Gelbmann 1993, Paunzen 1994). Allerdings erlitt das Experiment durch den Verlust der Sonde MARS96 einen Fehlschlag.

Nicht nur IUE-Daten wurden von Institutsmitgliedern für ihre Forschungsarbeiten benutzt sondern auch solche von ROSAT (z.B.: K. G. Strassmeier gemeinsam mit F. Kuerster für Aktive Sterne) und HIPPARCOS (Paunzen, E., Weiss, W. W. Kuschnig, R., Handler, G. Strassmeier, K. G., North, P., Solano, E., Gelbmann, M., Kuenzli, M., Garrido, R. 1998). Auch die Raumfahrt unterstützende Arbeiten wurden am Institut geleistet. Beispielsweise bestimmte A. Schnell 1986 mit dem 60" Telskop des L. Figl-Observatoriums im Rahmen der International Halley Watch Positionen des Kometen für eine genaue Bahnbestimmung vor der Landung der Raumsonden von ESA, Japan und der Sowjetunion auf diesem Himmelskörper. M. Ploner beobachtete im Rahmen seiner Dissertation 1993 geostationäre Satelliten zur Herleitung von Bahnkorrekturen.

Bis in die Gegenwart reichende Arbeiten betreffen COROT, MOST, HERSCHEL, ASTROVIRTEL und BRITE. COROT (COnvection, Rotation and planetary Transits) dient hauptsächlich der Untersuchung von Sternoszillationen und der Suche nach extrasolaren Planeten, MOST (Microvariability and Oscillations of Stars) zur Messung geringfügiger Helligkeitsschwankungen von Sternen, die von der Erde aus nicht messbar sind. Zur Unterstützung dieser photometrischen Satellitenexperimente wurde die Datenbank VISAT entwickelt. Für MOST (Start 2003) wurde am Institut in Wien eine Bodenstation errichtet. HERSCHEL wird mit einem 3,5-m-Spiegelteleskop ausgestattet im infraroten Spektralbereich beobachten. Der Wiener Beitrag wird im Rahmen eines europäischen Konsortiums für das Instrument PACS (Photodetector Array 


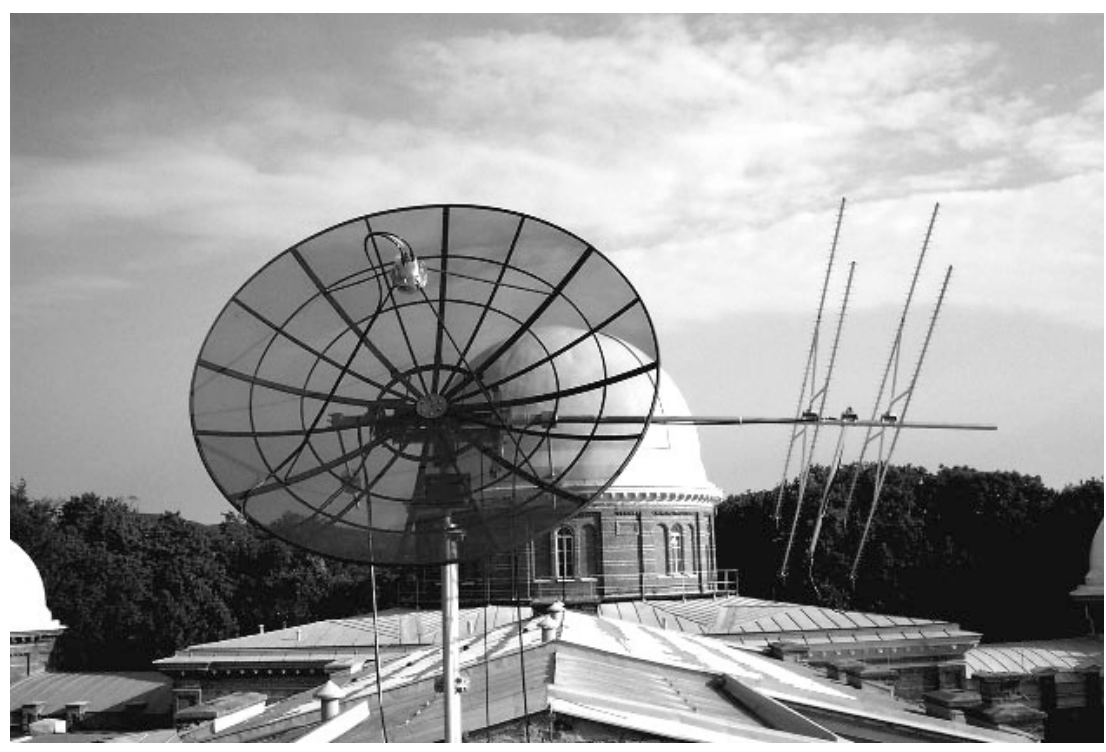

Abb. 2: Satelliten-Bodenstation Wien

Camera and Spectrograph) geleistet und von F. Kerschbaum betreut. Auf dem anderen Ende der Größenskala für Satellitenprojekte ist BRITE zu finden, ein im Weltraum stabilisierter Nanosatellit zur Messung von Sternoszillationen.

ASTROVIRTEL (Accessing Astronomical Archives as Virtual Telescopes) benützte als Vorläufer des "Virtual Observatory" Datenarchive als virtuelle Teleskope.

Trotz der vielfältigen Aktivitäten von Wiener Institutsmitgliedern wurde erst 1995 jemand aus Österreich in die Astronomy Working der ESA aufgenommen. Es war W.W. Weiss; ihm folgten F. Kerschbaum und W. Zeilinger. Neben R. Albrecht und $H$. Jenkner arbeiten 6 weitere Absolventinnen und Absolventen des Wiener Instituts im Ausland auf dem Gebiet der Weltraumastronomie.

Finanzielle Unterstützung wurde durch das Bundesministerium für Wissenschaft und Forschung, das Bundesministerium für Verkehr, Innovation und Technologie, das Bundesministerium für Wirtschaft und Arbeit, die Österreichische Akademie der Wissenschaften, die Austrian Solar and Space Agency bzw. die Austrian Space Agency und den Fonds zur Förderung der wissenschaftlichen Forschung gewährt. 


\section{Literatur}

Allgemein: Jahresberichte der Universitäts-Sternwarte Wien in den Mitteilungen der Astronomischen Gesellschaft sowie die Seiten einzelner Projekte auf der Instituts-Homepage (http://www.univie.ac.at/astro/ - Stand März 2008)

Baglin, A., Weiss, W. W., Bisnovatyi-Kogan, G. 1993: E.V.R.I.S on board MARS 94: the first space experiment devoted to stellar seismology in: Weiss, W. W., Baglin, A. (eds), Proc. IAU C oll. 137 "Inside the Stars", Astr. Soc. Pac. Conf. Ser. 40, 758

Ferrari d'Occhieppo, K. 1977: Josef Hopmann (mit Schriftenverzeichnis), Almanach ÖAW 126, 518

Gelbmann M.J. 1993: Simulation von photometrischen Effekten durch Satellitendrift, Diplomarbeit Univ. Wien

Haupt, H. 1996: Guntram Schutka, Mitt. Astron. Ges. 79, 9

Paunzen, E 1994: Die Simulation der Driftefekte beim Projekt EVRIS, Diplomarbeit Univ. Wien

Paunzen, E., Weiss, W. W., Kuschnig, R., Handler, G., Strassmeier, K. G., North, P., Solano, E., Gelbmann, M., Kuenzli, M., Garrido, R.: Pulsation in lambda Bootis stars, Astron. Astrophys. 335, 533, 1998

Prochazka, F.V., Tucker, R.H. (eds.):Modern Astrometry, Proc. IAU Coll. No.48, Wien 1978

Rakos, K.D. 1975,

Rakos, K.D., Kamperman, T.M. 1977, Astron.Astrophys. 55, 53

Schrutka, G. 1958: Mitt. Univ. Stwte. Wien 9, 251

Swaton G. 1965: Ableitung relativer Mondhöhen aus dem "PhotographicLunar Atlas" von Kuiper, Diss. Univ. Wien

Weiss, W.W., Kreidl, T.J. 1978: Ap-Stars in the Infrared, Wien 1978

Weiss, W.W., Schnell, A., Albrecht, R., Jenkner, H., Maitzen, H.M., Rakos, K., Mondre, E. (eds.): IUE Data Reduction, Wien, 1980 
